Extended Steady-State and High-Beta Regimes of Net-Current Free Heliotron Plasmas in the Large Helical Device

\author{
O. Motojima et al.
}




\title{
Extended Steady-State and High-Beta Regimes of Net-Current Free Heliotron Plasmas in the Large Helical Device
}

O.Motojima 1), H.Yamada 1), A.Komori 1), N.Ohyabu 1), O.Kaneko 1), K.Kawahata 1), T.Mito 1), T.Mutoh 1), K.Ida 1), S.Imagawa 1), Y.Nagayama 1), T.Shimozuma 1), K.Y.Watanabe 1), S.Masuzaki 1), J.Miyazawa 1), T.Morisaki 1), S.Morita 1), S.Ohdachi 1), N.Ohno 2), K.Saito 1), S.Sakakibara 1), Y.Takeiri 1), N.Tamura 1), K.Toi 1), M.Tokitani 3), M.Yokoyama 1), M.Yoshinuma 1), K.Ikeda 1), A.Isayama 4), K.Ishii 5), S.Kubo 1), S.Murakami 6), K.Nagasaki 7), T.Seki 1), K.Takahata 1), H.Takenaga 4), and

LHD Experimental Group

1) National Institute for Fusion Science, Toki, Gifu, Japan

2) EcoTopia Science Institute, Nagoya Univ., Nagoya, Aichi, Japan

3) Research Institute of Applied Machanics, Kyushu Univ., Kasuga, Fukuoka, Japan

4) Naka Fusion Institute, Japan Atomic Energy Agency, Naka, Ibaraki, Japan

5) Plasma Research Center, Tsukuba Univ., Tsukuba, Ibaraki, Japan

6) Department of Engineering, Kyoto Univ., Kyoto, Japan

7) Institute of Advanced Energy, Kyoto Univ., Uji, Kyoto, Japan

e-mail contact of main author motojima@LHD.nifs.ac.jp

\begin{abstract}
The performance of net-current free Heliotron plasmas has been developed by findings of innovative operational scenarios in conjunction with an upgrade of the heating power and the pumping/fueling capability in the Large Helical Device (LHD). Consequently, the operational regime has been extended, in particular, with regard to high density, long pulse length and high beta. Diversified studies in LHD have elucidated the advantages of net-current free heliotron plasmas. In particular, an Internal Diffusion Barrier (IDB) by combination of efficient pumping of the local island divertor function and core fueling by pellet injection has realized a super dense core as high as $5 \times 10^{20} \mathrm{~m}^{-3}$, which stimulates an attractive super dense core reactor. Achievements of a volume averaged beta of $4.5 \%$ and a discharge duration of 54-min. with a total input energy of $1.6 \mathrm{GJ}$ (490 kW in average) are also highlighted. The progress of LHD experiments in these two years is overviewed with highlighting IDB, high $\beta$ and long pulse.
\end{abstract}

\section{Introduction}

Fusion research is approaching a new era of burning plasmas. While the ITER project will play the most significant role in the new era, the necessity of basic research activities and capabilities which will not be covered by ITER has been discussed and a broader approach has been addressed. Although the broader approach is primarily defined based on a strategy aimed at a tokamak reactor, the fundamental physical elements, i.e., steady state operation, advanced plasma regimes emphasizing high- $\beta$ and control of power fluxes to walls are basic requisites not specific to tokamaks. Indeed, the Large Helical Device (LHD) project was developed to address these significant elements a decade ago [1] and has been conducted to resolve these three core issues in a different manner from that of tokamaks [2]. The core competency of LHD lies in its intrinsic capability of steady-state net-current free plasmas. Thorough exploration in LHD should lead to the establishment of not only a prospect for a helical fusion reactor but also to a comprehensive understanding of toroidal plasmas.

LHD is the world's largest superconducting magnetic confinement device, which employs a heliotron configuration. A nominal plasma volume of $30 \mathrm{~m}^{3}$ results from the major and minor radii of $3.7 \mathrm{~m}$ and $0.64 \mathrm{~m}$, respectively. A confining magnetic field of up to $3 \mathrm{~T}$ is provided in 
complete steady-state by means of the superconducting coil system. The current heating capability is $16 \mathrm{MW}$ of NBI, $2.9 \mathrm{MW}$ of ICRF and 2.1 MW of ECH. In particular, ICRF and ECH facilities have an engineering capability of steady state operation with $1 \mathrm{MW}$. LHD is equipped with two different divertor systems; the Helical Divertor (HD) and the Local Island Divertor (LID) [3]. The HD has a shape similar to the double-null open divertor in tokamaks except for its helically twisted structure. The LID uses an $m / n=1 / 1$ magnetic island controlled by the externally applied resonant field to guide particle and heat fluxes to the divertor plates.

The plasma parameters as well as physical understanding have been progressing steadily since the last IAEA Fusion Energy Conference in 2004 [2]. The most highlighted achievement in the latest experimental campaign is the discovery of a Super-Dense-Core plasma operation created by an Internal Diffusion Barrier (IDB) [4]. A synergetic effect of highly efficient pumping by means of the LID and core fueling by repetitive pellet injection generates the IDB with a central density of $5 \times 10^{20} \mathrm{~m}^{-3}$. A steep gradient in the density profile is formed by a drastic improvement in particle transport, i.e., IDB. It has been said that a helical plasma has an advantage of high density operation and the discovery of the IDB emphasizes this advantage much more than expected before and stimulates development of an attractive reactor operation with high density and relatively low temperature. Simultaneously, the IDB is attracting keen interest into the physics of its transition and structural formation.

Long pulse operation by means of ion cyclotron resonant heating (ICRH) [5] has been prolonged to close to one hour (precisely $3268 \mathrm{~s}$ ) with a heating power of $500 \mathrm{~kW}$. The total input energy for this discharge reached 1.6 GJ which is a world record in magnetic confinement experiments. Investigation of highly energetic particles and control of the heat load on the divertor plates have made a significant contribution to this achievement. High $\beta$ discharges also have been advanced by exploration of aspect-ratio control [6] and consequently a beta of $4.5 \%$ has been achieved. The understanding of pressure driven MHD modes has been deepened in the high $\beta$ regime approaching the key level of $5 \%$ for a reactor.

In addition to these major achievements, important progress has been made in various areas in these two years. Characterization of edge plasmas is taken up for the discussion of particle control, effect on wall materials, properties of turbulence and the edge transport barrier. Transient heat transport in the core plasmas has been investigated to clarify the nonlinear, non-local and off-diagonal nature of turbulent transport. These results are reported in individual contributions to this conference. In this overview, three major topical results, i.e, IDB, high $\beta$, long pulse operation, and their related topics are highlighted.

\section{Parameter Extension}

Before describing each topical result, the

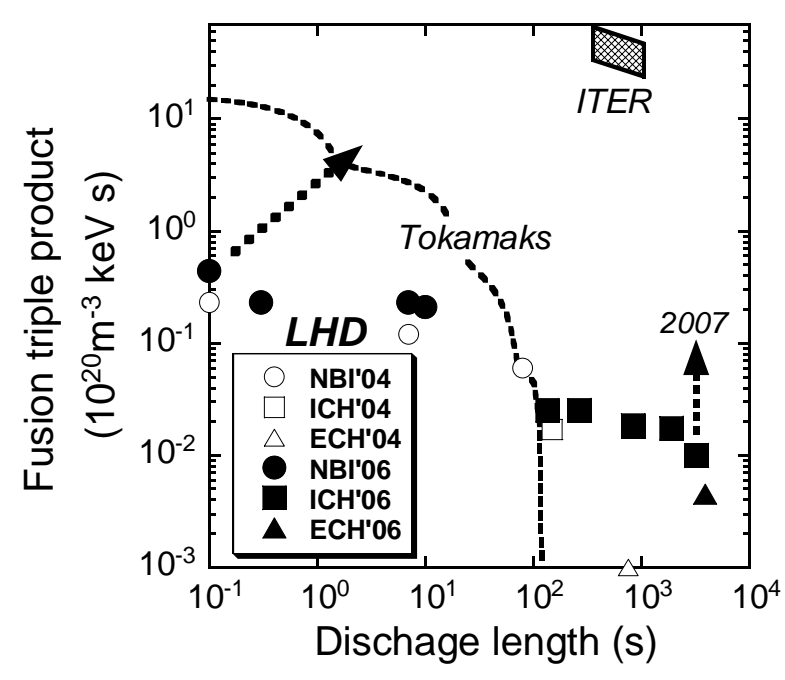

FIG.1 Achieved operational regime in the plane of fusion triple product and discharge length. A dotted curve shows the envelope of tokamaks. Open and closed symbols denote the presented data in FEC 2004 and 2006, respectively. 
latest parameter extension is summarized in this section. These achievements are definitely consequences from discoveries of innovative experimental scenarios. Figure 1 shows the operational regime in terms of the fusion triple product and the duration time of plasma discharges. LHD has already provided a unique operational regime in long pulse operation. The performance of the fusion triple product has also been extended in these two years. The highest $n \tau_{\mathrm{E}} T$ has been realized by the IDB despite about a $40 \%$ reduction in the effective confinement volume due to the LID configuration compared with the standard magnetic configuration with $R_{a x}=3.6 \mathrm{~m}$. The characterization of high performance plasmas with IDB will be described in Sec.3. Tolerance against the interchange modes which has been observed in earlier works can be maintained in the higher aspect ratio configuration with worse stability but better confinement and power deposition. Optimization of the compromise between confinement and MHD stability has led to a $\beta$ of $4.5 \%$ which is maintained for more than 10 times of the energy confinement time. This study will be discussed in Sec.4. Handling of the heat load, in particular, onto the divertor tiles is a critical issue. The intrinsic character of a built-in divertor has a remarkable feature that a tiny change of the magnetic axis position results in a wide dispersion of heat flux onto the divertor plates. Dynamical slow variation of the magnetic axis position by only $3 \mathrm{~cm}$ has demonstrated saturation of the temperature rise of the divertor tiles below a tolerable temperature with steady-state heating of $0.68 \mathrm{MW}$. This subject will be discussed in Sec.5. The ion temperature is raised to $13.5 \mathrm{keV}$ by applying heavier gas, argon, instead of hydrogen. This scenario is attributed to the enhancement of ion heating under the condition of an NBI energy as high as $180 \mathrm{keV}$ which primarily provides electron heating in a hydrogen operation. This result has shown that the increase in ion heating increases ion temperature, which strongly motivates the upgrade of the NBI with a lower energy.

\begin{tabular}{llcccc}
\hline & & high $n \tau_{\mathrm{E}} T$ & high $\beta$ & long pulse & high $T_{i}$ \\
\hline$T_{e}(0)$ & $(\mathrm{keV})$ & 0.85 & 0.4 & 2.0 & 4.5 \\
$T_{i}(0)$ & $(\mathrm{keV})$ & 0.85 & - & 2.0 & 13.5 \\
$n_{e}(0)$ & $\left(10^{19} \mathrm{~m}^{-3}\right)$ & 46 & 2.8 & 1.2 & 0.35 \\
$\bar{n}_{e}$ & $\left(10^{19} \mathrm{~m}^{-3}\right)$ & 21 & 2.7 & 0.8 & 0.3 \\
$\tau_{\mathrm{E}}$ & $(\mathrm{s})$ & 0.11 & 0.013 & 0.08 & 0.066 \\
$\beta$ & $(\%)$ & 1.5 & 4.5 & 0.05 & 0.3 \\
$W_{p}$ & $(\mathrm{~kJ})$ & 1100 & 81 & 50 & 235 \\
$P_{a b s}$ & $(\mathrm{MW})$ & 10 & 6.4 & 0.68 & 3.6 \\
$R_{a x}$ & $(\mathrm{~m})$ & 3.75 & 3.6 & $3.67-3.7$ & 3.7 \\
$B$ & $(\mathrm{~T})$ & 2.64 & 0.45 & 2.75 & 2.68 \\
Remarks & IDB with LID & $A_{p}=6.6$ & 31 min. & Ar puff \\
\hline
\end{tabular}

Table I Achieved parameters in LHD

\section{Internal Diffusion Barrier Leading to Super Dense Core and Consequent Innovative Scenario of Super Dense Core Reactor}

The LID concept has demonstrated its basic divertor function of efficient particle control. In the pellet fuelled plasmas in the LID configuration, a new type of confinement improvement in the core region has been discovered. An IDB develops naturally in NBI heated plasmas in LHD as a peaked, high density profile is generated by multiple pellet injections from the outside mid-plane under powerful particle control using the LID. Typical waveforms are 
illustrated in Fig.2.

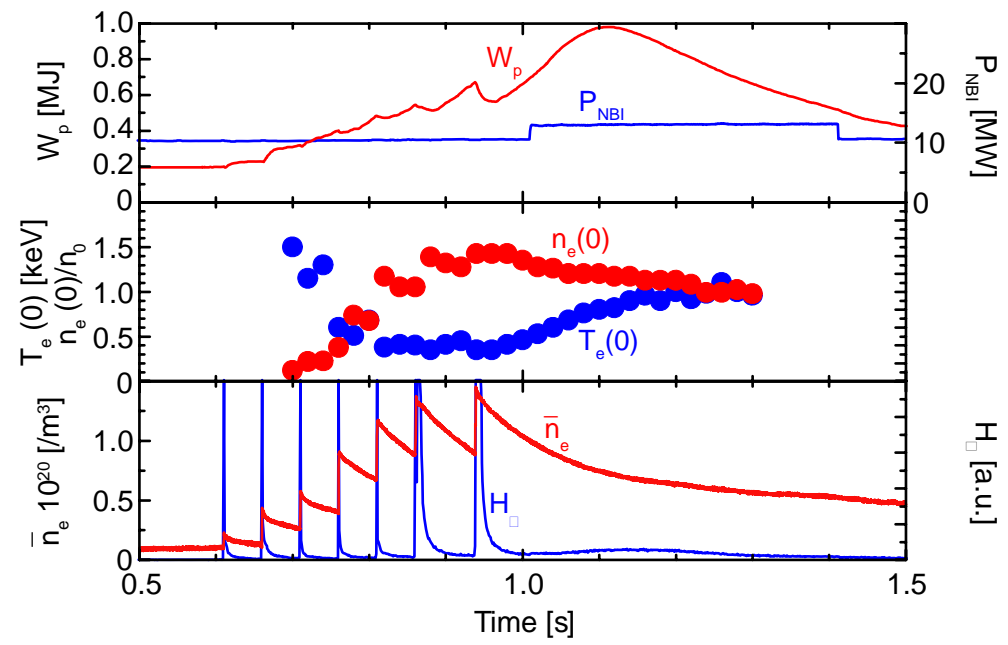

FIG.2 Waveforms of a typical discharge generating IDB.

The density and temperature profiles are shown in Fig. 3 for a typical plasma with IDB $\left(R_{a x}\right.$ $($ vacuum magnetic axis $)=3.75 \mathrm{~m}, B($ magnetic field $)=2.64 \mathrm{~T}, P_{a b s}($ absorbed NBI power $)=$ $10 \mathrm{MW}$ ). They are measured by a Thomson scattering diagnostic along the major radius $R$, where the plasma is horizontally elongated. The electron density in a core region has reached $5 \times 10^{20} \mathrm{~m}^{-3}$. The peaked density profile has a sharp jump in the density gradient located in narrow range of normalized minor radius $0.3<\rho<0.5$, and after the termination of a sequence of pellet injections, the central density decays slowly with a time constant of about 1s. These observations show the spontaneous generation of the IDB. The radial width of the IDB is about $0.1 \mathrm{~m}(\Delta \rho \sim 0.2)$. The density gradient outside the IDB is $1 / 7$ of that in the IDB with $n_{e} \sim 6 \times 10^{19} \mathrm{~m}^{-3}$ at the last closed flux surface $(\rho=1)$. In contrast to the density profile and unlike the tokamak ITB (internal transport barrier) discharges, there is no increase in the temperature gradient, possibly because the density becomes extremely large in LHD. Inside the IDB region, the density and temperature gradients are nearly zero. Despite an extremely high density, the central electron temperature is still $0.85 \mathrm{keV}$, which realizes one atmospheric pressure. The plasmas with the IDB exhibit the highest fusion plasma performance achieved so far on LHD, $n(0) \tau_{\mathrm{E}} T(0) \sim 4.4 \times 10^{19} \mathrm{keVm}^{-3} \mathrm{~s}$, despite about a $40 \%$ reduction in effective

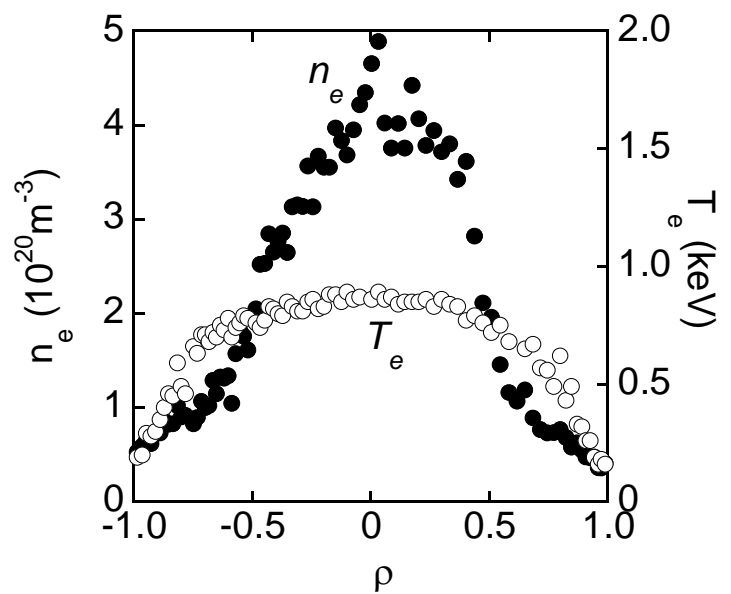

FIG.3 Density and temperature profiles of a discharge with the IDB in the case with $R_{a x}$ $=3.75 \mathrm{~m}$.

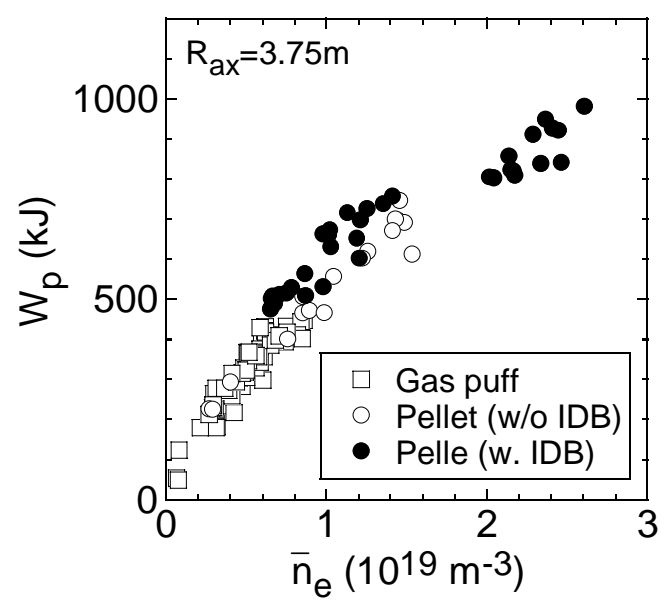

FIG.4 Store energy evolution with the density. 
confinement volume (due to the LID) from standard HD discharges. Repetitive pellet injection can maintain the IDB for $1 \mathrm{~s}$ which is more than 10 times the energy confinement time. The volume averaged beta and central beta have reached $1.5 \%$ and $4.4 \%$ despite the high magnetic field. The central beta exceeding $5 \%$ has been achieved when the magnetic field is lowered to $1.5 \mathrm{~T}$.

The energy confinement in helical systems shows a clear density dependence in the moderate density regime. The degradation of the energy confinement time in the high density region and extension of a density dependent regime by pellet injection are also observed. This plasma with the IDB has shown more distinguished improvement than pellet fueled discharges reported in earlier works [7] (see Fig.4). Figure 5 shows the comparison of the energy confinement time in the LID experiment with the prediction from the empirical scaling, ISS95 [8]. A good IDB can be realized in the case with $R_{a x}=3.75 \mathrm{~m}$. An earlier confinement study has indicated that this configuration has worse confinement than the case with $R_{a x}=3.6 \mathrm{~m}$ under standard HD operation. Nonetheless, the confinement enhancement of the plasmas with the IDB on the scaling is close to the case with $R_{a x}=3.6 \mathrm{~m}$ with the moderate density and the state with good confinement has been extended to the extremely high density regime by the formation of the IDB.

The temperature gradient in the outer region is determined by anomalous thermal diffusivity. Outside of the IDB, the density is suppressed by powerful pumping by the LID. This situation is favorable to enhancement of the temperature. The $\nabla T_{e}$ in edge increases by lowering the edge density. Also the central electron temperature is enhanced simultaneously.

The performance improvement by the IDB depends on the magnetic axis position: $R_{a x}$ in a different way from the clear dependence of the magnetic axis position on energy confinement [9]. It is partly attributed to the power deposition of the NBI. The NBI tangency radius is 3.70 $\mathrm{m}$; thus, the power deposited in the core $(\rho<0.5)$ for $R_{a x}=3.65 \mathrm{~m}$ is substantially lower than that for $R_{a x}=3.75 \mathrm{~m}$. A more important point for determining the performance is the minor

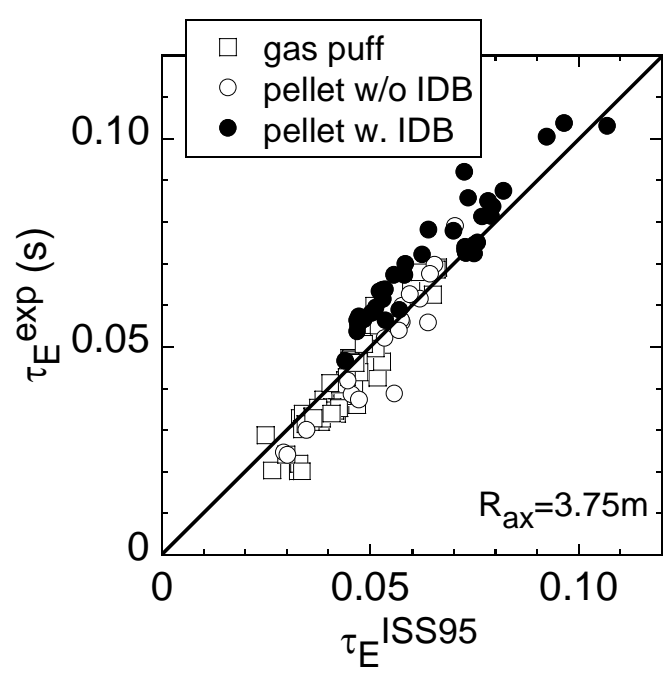

FIG.5 Comparison of the energy confinement time in the LID experiments with the prediction from the ISS95 scaling.

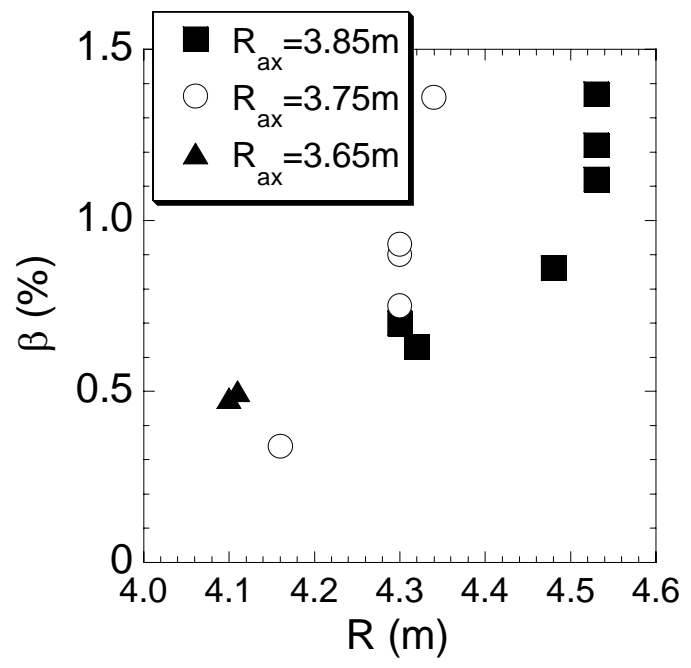

FIG.6 With increasing $\beta$ and $R_{a x}$, the foot location of the IDB $\left(R_{\text {foot }}\right)$ increases. The red, blue and green curves are $R_{s p}$ for $R_{a x}=3.85$, $3.75,3.65 \mathrm{~m}$, respectively. 
radial extent defined by the IDB foot (jump in $\nabla n)$ location $\left(R_{f o o t}\right)$, which increases with $R_{a x}$ and $\beta$. Figure 6 shows $R_{\text {foot }}$ as a function of the average beta for three different configurations $\left(R_{a x}=3.65 \mathrm{~m}, 3.75 \mathrm{~m}, 3.85 \mathrm{~m}\right)$. The "standard" configuration $\left(R_{a x}=3.75 \mathrm{~m}\right)$ with $\beta(0)=4.4 \%$, and the IDB foot at $\rho=0.56$ yields optimum performance. For the outward shifted configuration $\left(R_{a x}=3.85 \mathrm{~m}\right)$, the radius of the IDB foot grows with increasing $\beta$ and is close to the last closed surface at $\beta=1.38 \%$. For the inward shifted case $\left(R_{a x}=3.65 \mathrm{~m}\right)$, the high density region is less distinguished, with the IDB foot at $\rho=0.45$ and $\langle\beta\rangle=0.5 \%$. The mechanism to determine the position of the IDB foot attracts physical interest as well as further performance improvement, i.e., extension of the region defined by the IDB. There exist correlations between $R_{f o o t}$, and the reversal of the sign of magnetic shear and the boundary of the magnetic well and hill. A more accurate and detailed equilibrium description and clarification of causal and resultant phenomena are required to clarify the deterministic mechanism of the position of $R_{\text {foot }}$.

The density limit in helical systems is quite different from that in tokamak. The Sudo scaling [10] defined by $n_{e}^{\text {Sudo }}\left(10^{20} \mathrm{~m}^{-3}\right)=0.25\left(P_{a b s} B /\left(a^{2} R\right)\right)^{0.5}$, where $P_{a b s}(\mathrm{MW})$, has been often used to discuss the operational density limit in helical systems. This scaling is based on the power balance of the heating power and the radiation loss which is proportional to $n_{e}{ }^{2}$. A systematic study on density limit considering profiles in LHD suggests that the density where the temperature drops to $100 \mathrm{eV}$ in the edge region is a decisive parameter. The electron temperature at $\rho=1$ is well fitted by $\left(P_{\text {tot }}^{0.5} / n_{e}\right)^{2 / 3}$, as is shown in Fig. 7. Significant deviation from this fitting is triggered at $T_{e}<100 \mathrm{eV}$, suggesting that the heating power is lost by enhanced radiation in the low temperature regime, and/or the transport property changes. The reason why this occurs at $T_{e} \sim 100 \mathrm{eV}$ has not been clarified yet, nonetheless, it can be postulated that transition of physical properties playing a deterministic role in density limit occurs in the vicinity of $T_{e}$ of $100 \mathrm{eV}$. In the case with the LID configuration, low edge density realized by the pumping function of the LID is advantageous in avoiding the radiative collapse which determines the operational density limit and hence leads to higher core density. Figure 8 shows the plots of the central density normalized by the density at the temperature of $100 \mathrm{eV}$, which corresponds to the peakedness of the density profile, as a function of the

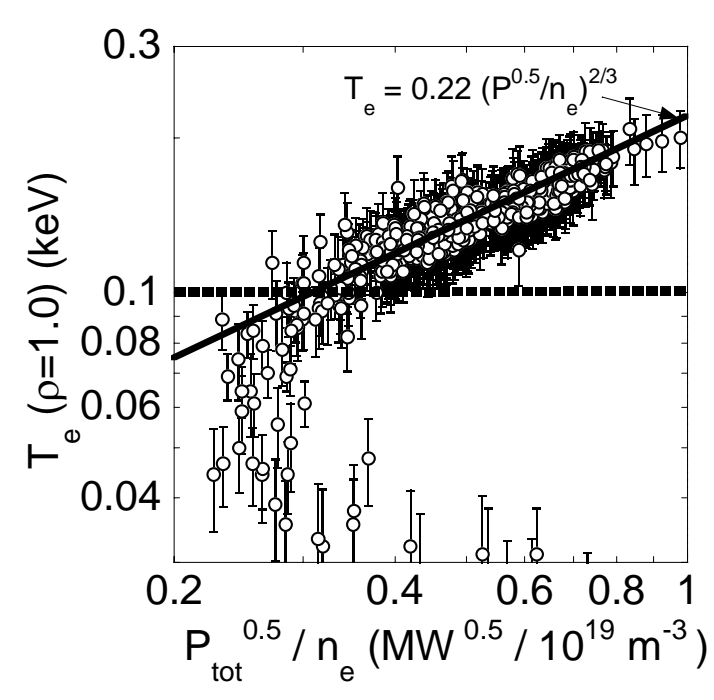

FIG.7 Electron temperature dependence on the square root of the heating power divided by the local electron density at the last closed flux surface.

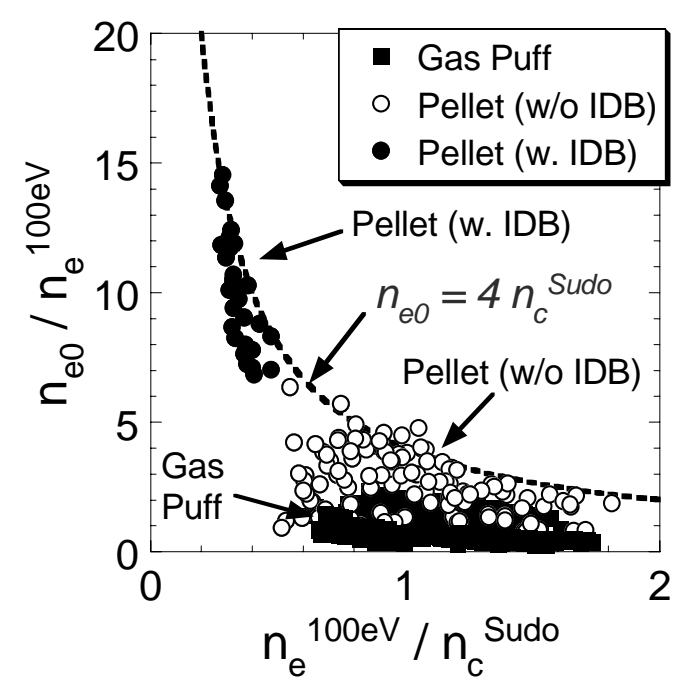

FIG.8 Density regime of high density oriented discharges. 
density at the temperature of $100 \mathrm{eV}$ normalized by the Sudo density limit. Pellet injection leads to the peaked density profile and the formation of the IDB greatly enhances the peakedness. While the central density when the IDB is created has reached four times the Sudo density, the edge density at the temperature of $100 \mathrm{eV}$ is well below the high density discharges with a normal pellet as well as with gas puffing. This means that a large margin against the density limit still exists in the high density plasmas with the IDB.

These results of the plasma with high density core generated by the IDB suggest a novel fusion ignition scenario which enables operation at very high density and relatively low temperature. Such a scheme is particularly attractive for helical devices because (a) they do not require current drive (which is most effective at low density), (b) operation at high collisionality reduces the effect of the helical ripple diffusion regime in which energy and particle losses increase with decreasing collision frequency (increasing temperature), (c) large extension of operational density mitigates plasma wall interaction (reduction of erosion, etc.), which reduces engineering demand of a reactor.

\section{MHD characteristics towards the High Beta Regime Approaching the Key Level of $5 \%$}

Since the helical coil of LHD consists of 3 independent layers in the minor radial direction, the plasma aspect ratio, $A_{p}$, can be controlled by changing the current in each layer. Large $A_{p}$ reduces the Shafranov shift and is preferable with regard to the MHD equilibrium beta limit while MHD stability is violated due to suppression of the spontaneous generation of a magnetic well. It has been recognized that turbulent transport as well as neoclassical transport degrades with the shift of the magnetic axis. The NBI heat deposition also deteriorates with the Shafranov shift through the enhancement of the orbit loss of trapped particles, in particular, at the low magnetic field where the high beta experiments are carried out. Earlier works have indicated that the tolerance against the interchange mode is larger than the Mercier criterion and that the core region is stabilized by the spontaneous generation of a magnetic well [11]. Characteristics of MHD instability observed in the experiment and their comparison with advanced theories have indicated the important roles of the modulation of the plasma boundary and compressibility to mitigate the deterioration of confinement performance due to pressure driven instabilities [12]. In regular operation $\left(A_{p}=5.8\right)$, disruptive phenomena have not been observed, however, the minor and local relaxation occurs when the ideal stability is violated significantly due to a large pressure gradient and the magnetic fluctuation amplitude due to the resistive interchange mode increases in the peripheral region as beta increases. Therefore, the compromise between the confinement related advantage and the effect of MHD instability maximizes the achievable beta. This optimization of the

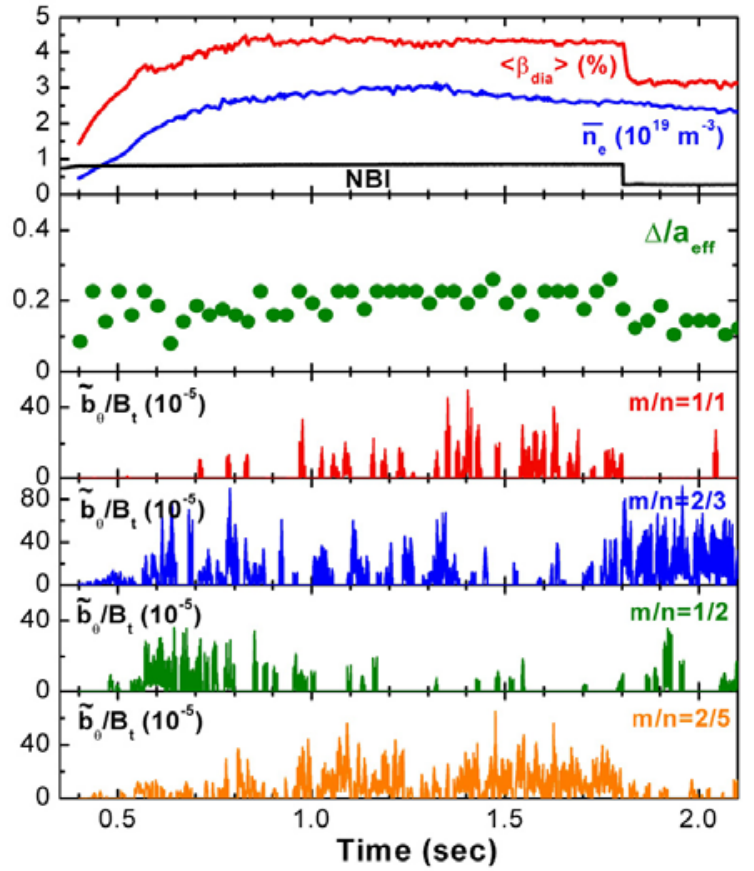

FIG.9 Typical waveforms and MHD activities in a high- $\beta$ discharge. 
aspect ratio is analogous to the maximization of beta through the magnetic axis scan.

As a consequence of a series of systematic studies changing the aspect ratio and the magnetic field, the highest beta value of $4.5 \%$ has been achieved at $A_{p}=6.6$ and the magnetic field $B$ of $0.425 \mathrm{~T}$. Figure 9 shows the highest- $\beta$ discharge with $\left\langle\beta_{\mathrm{dia}}>=4.5 \%\right.$. The $R_{a x}$ is set at $3.6 \mathrm{~m}$. The target plasma is produced and maintained by injecting two co- neutral beams and a counter one. The $<\beta_{\text {dia }}>$ increases with $\bar{n}_{e}$ by gas puffing and reaches $4.5 \%$ at $0.89 \mathrm{~s}$. The gas puff is turned off at $1.35 \mathrm{~s}$, whereas the state with $\left\langle\beta_{\mathrm{dia}}>>4 \%\right.$ is maintained for $10 \tau_{\mathrm{E}}$ or more until two neutral beams are turned off at $1.8 \mathrm{~s}$. The $R_{a x}$ was identified by an electron temperature profile measured with the Thomson scattering system. The Shafranov shift, $\Delta / a_{\text {eff, }}$, is suppressed to less than 0.25 in the discharge, where $\Delta$ is defined as $\Delta=R_{a x}-R_{00}$ and $R_{00}$ is the central position of the last closed flux surface and the Shafranov shift is much below the equilibrium beta-limit defined as $\Delta / a_{\text {eff }}=1 / 2$. Peripheral MHD activities such as $m / n=1 / 1$, $2 / 3,1 / 2$ and $2 / 5$ are dominantly observed during the discharge, and they rotate with $f<15 \mathrm{kHz}$ in the electron diamagnetic direction. The MHD modes with $m / n>1$ which have a resonance in the core have not been observed except for at the beginning of the discharge since the magnetic well formation and the elimination of resonances due to the increase of the rotational transform are completed in the core region in the quite early phase. The MHD modes with $m / n<1$ which have resonances in the periphery, in particular the $m / n=2 / 3$ and $1 / 2$ modes are excited in the early phase and restrain the increase of $\left\langle\beta_{\mathrm{dia}}>\right.$. When $\left\langle\beta_{\mathrm{dia}}>\right.$ exceeds around $4 \%$, the amplitudes of these modes gradually decrease and become interrupted. The activity is intermittent or even disappears. Although the amplitude of the $\mathrm{m} / \mathrm{n}$ $=2 / 5$ mode increases, it is relatively small compared with other modes. The tendency that MHD modes are suppressed starting with a mode with the resonance in the inner region is a common feature in the high-beta regime. This phenomenon is accompanied by local pressure profile flattening in the vicinity of the resonances. The effect of the observed peripheral MHD modes is not crucial but not negligible for the production of high-beta plasma in the $\left\langle\beta_{\mathrm{dia}}>\right.$ range of $>$ $4 \%$. According to linear MHD analysis, the Mercier criterion $D_{I}$ on the $t \geq 1$ surface is not violated (less than 0.2 ) even in the highest $\beta$ plasma. Strong magnetic shear secures the stability even in the magnetic hill. Therefore the observed MHD modes in the magnetic hill region are resistive interchange modes.

Although the MHD instability is not crucial when $A_{p}$ is less than 7, the MHD instabilities limiting the achievable beta are observed in the aspect ratio larger than 7 . An increase of the aspect ratio leads to an increase of rotational transform and then the resonance layer shifts to the core region where the magnetic shear is weak. When $A_{p}$
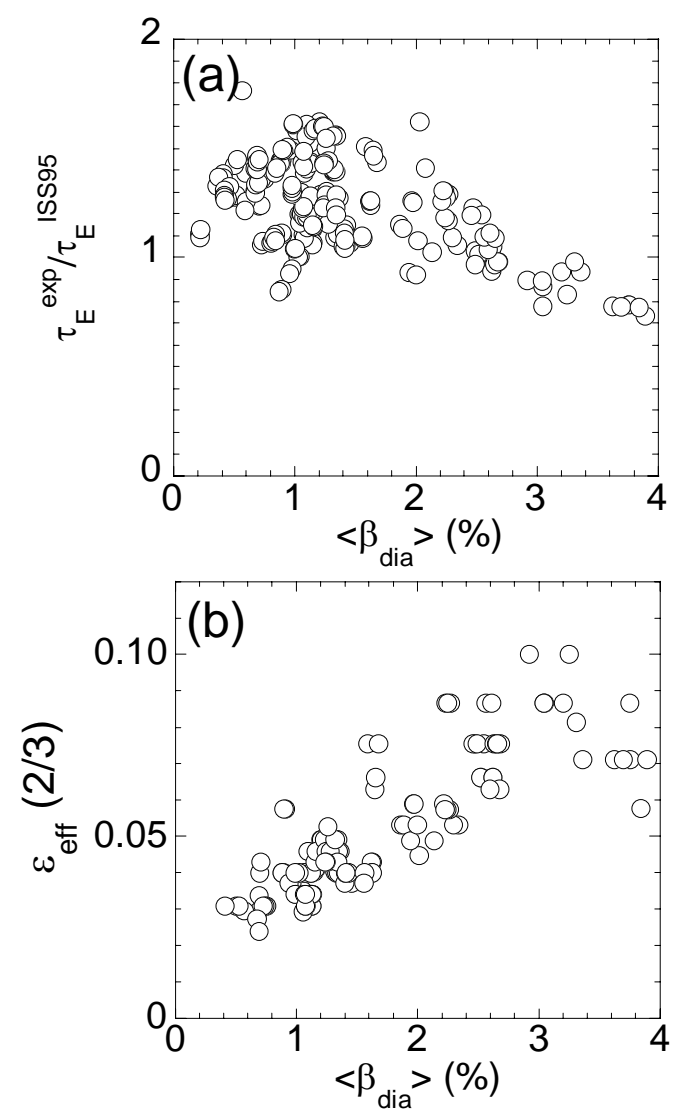

FIG.10 Enhancement factor of the energy confinement time on the ISS95 scaling and the effective helical ripples at $\rho=2 / 3$ as a function of the beta in the configuration with $R_{a x}=3.6 \mathrm{~m}$, $A_{p}=6.3$. 
exceeds 7 , the $m / n=1 / 1$ ideal stability is violated. Under this condition, the $m / n=1 / 1$ mode suddenly grows and leads to a minor collapse in the core region. Nonetheless, the plasma does not disrupt completely and the relaxed state with the large static $m / n=1 / 1$ island is maintained for much longer than the energy confinement time. At lower $A_{p}$, the achievable beta is reduced due to degradation of confinement and heat deposition. The empirical scalings $[8,13]$ of the energy confinement time are rephrased into the dependence of beta on the heating power like $\beta \propto P_{a b s}^{0.39-0.41}$. The beta value in the configuration with the maximum beta still increases with the heating power although confinement tends to decline as $\beta \propto P_{a b s}^{0.25}$ in the high beta region compared with the prediction by the scalings. This means that the achievable beta is limited by confinement, not by stability.

Figure 10(a) shows the confinement enhancement factor relative to the prediction from the scaling as a function of beta for the case with $A_{p}=6.3$ where a series of data close to $4 \%$ are available, although this is not the high beta optimized configuration. Above a $<\beta_{\mathrm{dia}}>$ of $1 \%$, a gradual degradation with $\left\langle\beta_{\mathrm{dia}}>\right.$ has been observed. There are two cause for this degradation, which cannot be discussed separately at present. One is the confinement degradation due to the increase of density, which has been widely observed in the experiment. Another is the increase in $\varepsilon_{\text {eff }}$ due to the Shafranov shift. Although the underlying physics has not been clarified yet, in LHD, anomalous transport as well as neoclassical transport is suppressed when $\varepsilon_{\text {eff }}$ is reduced [13]. Indeed, the evaluated $\varepsilon_{\text {eff }}$ at $\rho=2 / 3$ shows a correlation with the confinement degradation (see Fig.10(b)). This trend agrees with the envelope confirmed in the confinement study with the magnetic axis scan. Dynamical control of the magnetic axis position by the vertical field is in plan, which will resolve this issue.

\section{Long Pulse Operation and Solution of Issues towards Steady State}

Long pulse operation has been pursued to demonstrate the advantage of net-current free plasmas and the steady state operation regime has been greatly extended in LHD in these two years. A high-temperature plasma is created and maintained for more than 30 min with a world record injected heating energy of $1.3 \mathrm{GJ}$ in 2005 and recently for 54 min with $1.6 \mathrm{GJ}$ in 2006 experimental campaign. The long pulse operation is primarily sustained by the Ion Cyclotron Range Frequency (ICRF) heating. For steady-state operation of the LHD, ICRF antennas, i.e., six antenna loops are installed on the outboard side of the torus at the vertically
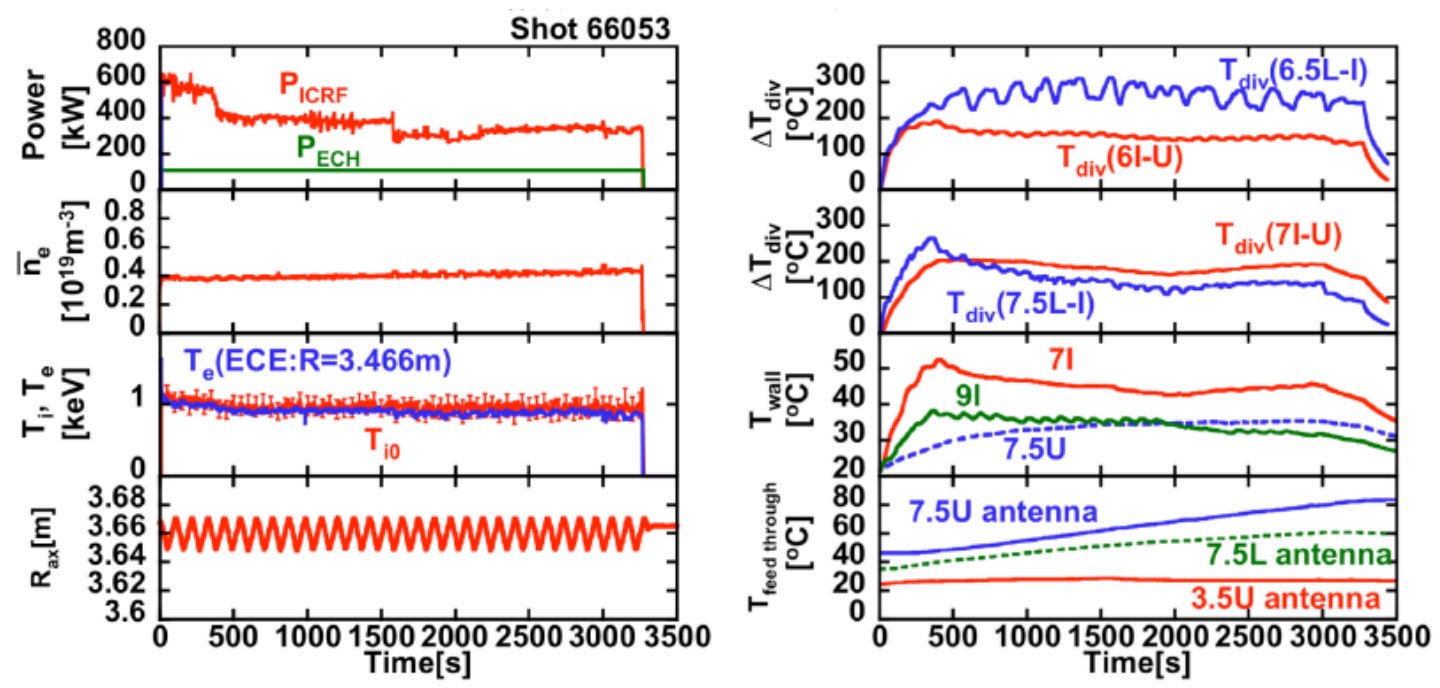

FIG.11 Waveforms of a 54-min. long discharge. 
elongated sections and four antennas are connected to transmitters through specially developed ceramic feed-throughs, coaxial lines, and liquid impedance tuners. The ICRF heating mode for the long-pulse experiment used minority heating with helium as the majority ions and hydrogen as the minority ions. In addition a continuous wave $(\mathrm{CW})$ Electron Cyclotron Heating $(\mathrm{ECH})$ system provides $110 \mathrm{~kW}$ in steady state. For the 30 min operation in 2005, repetitive NBI heating of $500 \mathrm{~kW}$ with $25 \mathrm{~s}$ pulses was also used as an auxiliary heating device. In this operation, the averaged input power was $680 \mathrm{~kW}$ and the input energy to the plasma was 1.3 GJ. Both ion and electron temperatures were kept at $2 \mathrm{keV}$ with the density of $7-8 \times 10^{18} \mathrm{~m}^{-3}$ for $30 \mathrm{~min}$.

In 2006, the steady state operation region was extended using ICRF and EC heating. A successful result has been achieved by controlling the input power level during the operation as a way to suppress the arcing which was the main cause of sudden impurity influx from the chamber wall or the divertor carbon plates. The temporal behavior of 54 minutes operation is shown in Figure 11. The plasma discharge is terminated by the loss of heating power due to an engineering control accident. The ICRF and ECH were injected continuously. The input heating power was gradually decreased and the average power was $380 \mathrm{~kW}$ for ICRF and 110 $\mathrm{kW}$ for $\mathrm{ECH}$. The total input energy to the plasma reached 1.6 GJ. The ICRF power was manually decreased to reduce occasional metal impurity influx. The line-averaged electron density was $0.4 \times 10^{19} \mathrm{~m}^{-3}$ and electron and ion temperatures were around 0.8 to $1 \mathrm{keV}$.

The magnetic axis position was swept back and forth between $3.65 \mathrm{~m}$ and $3.67 \mathrm{~m}$ in order to disperse the divertor heat load effectively. The three-dimensional heat-deposition profile of the LHD helical divertor was modified, and during long-pulse discharges it effectively dispersed the heat load using a magnetic-axis swing technique developed at the LHD. The measurement of Langmuir-probe arrays on the divetor tiles shows that the axis swing also dispersed the particle flux on each divertor tile in a direction perpendicular to the leg traces. A sweep of only $3 \mathrm{~cm}$ in the major radius of the magnetic axis position (less than $1 \%$ of the major radius of the LHD) was enough to disperse the divertor heat load. Eventually, the temperature of the divertor plates could be controlled below $300{ }^{\circ} \mathrm{C}$ in the steady state operation with the current heating power.

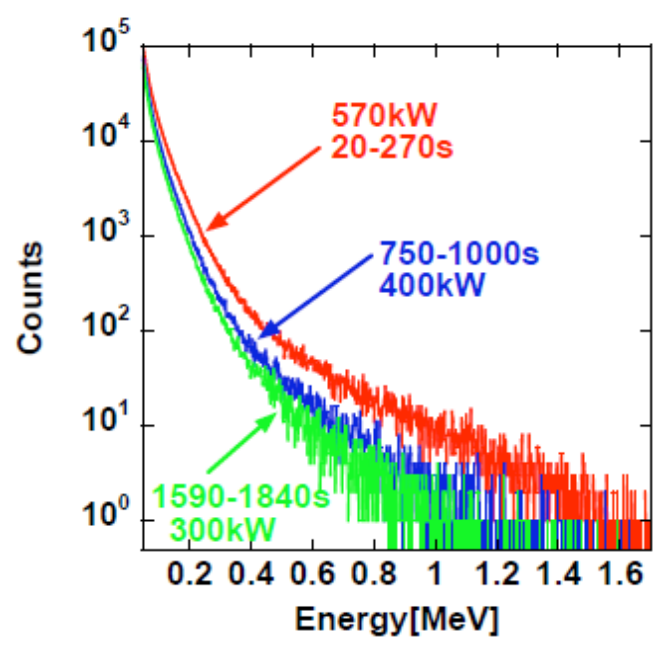

FIG.12 High energy ion measurement by CXNPA during 54 min operation. Flux counts are summed up during the indicated intervals.

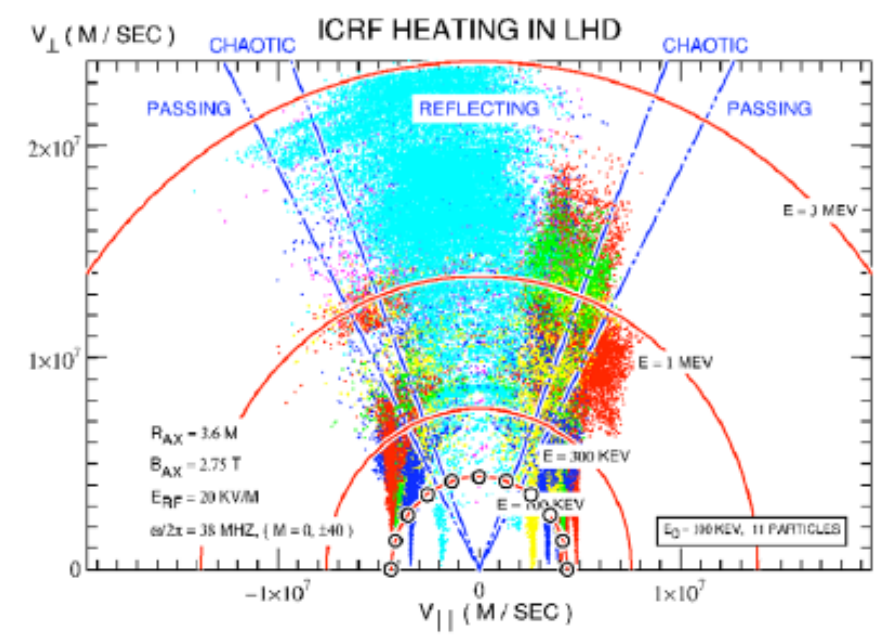

FIG.13 Poincare plots of calculated ICRF heated protons in the parallel and perpendicular velocity plane ( $R_{a x}=3.69 \mathrm{~m}, \mathrm{~B}=2.75 \mathrm{~T}, \mathrm{f}=38$ $\mathrm{MHz}$ ). 
A basic physical reason for the success of steady state operation is that highly energetic trapped ions are well confined due to drift optimization (configuration with the inward shifted magnetic axis). A high ion energy tail of up to $1.6 \mathrm{MeV}$ has been observed in long pulse discharges (see Fig.12). The orbit calculation in the LHD 3-D geometry including the ergodic layer surrounding the nested flux surfaces suggests that the energetic particle in the MeV range can be confined (see Fig.13). The confinement capability of highly energetic trapped particles has been demonstrated. Related $\alpha$-particle simulation experiment by using 2 nd harmonic ICRF heating confirms this scenario.

\section{Conclusions}

New findings overviewed in this paper have enabled us to extend the envelope of explorable physical parameter space in LHD. In particular, major progress in high density, long pulse length and high beta is highlighted. An Internal Diffusion Barrier (IDB) by combination of efficient pumping by the local island divertor function and core fueling by pellet injection has realized a super dense core as high as $5 \times 10^{20} \mathrm{~m}^{-3}$, which is exploring a new concept of a super dense core reactor with low temperature with a density more than $5 \times 10^{20} \mathrm{~m}^{-3}$ and a temperature less than $10 \mathrm{keV}$. A volume averaged beta of $4.5 \%$ have been maintained for more than 10 times the energy confinement time and discharge duration of 54-min. with a total input energy of $1.6 \mathrm{GJ}$ (490 kW in average) have been achieved on steady state basis. Other diversified studies in LHD have also elucidated the advantages of net-current free heliotron plasmas. The experiments in LHD have been offering a unique platform for physical studies of steady state operation and high beta. This progress shows that the heliotron configuration has a high potential as a steady-state fusion reactor.

\section{Acknowledgements}

The authors are grateful to contributions from all collaborators in universities and institutions all over the world. Tremendous efforts by the technical staff to operate LHD are greatly acknowledged.

\section{References}

[1] IIYOSHI, A., et al., Fusion Technol. 17 (1990) 169.

[2] MOTOJIMA, O., et al., Nucl. Fusion 45 (2005) S255.

[3] MORISAKI, T., et al., J. Nucl. Mater. 337-339 (2005) 154.

[4] OHYABU, N., et al., Phys.Rev.Lett. 97 (2006) 05502.

[5] MUTOH, T., et al., J.Plasma Fusion Res. 81 (2005) 229.

[6] WATANABE, K.Y., et al., Nucl. Fusion 45 (2005) 1247.

[7] SAKAMOTO, R., et al., Nucl.Fusion 41 (2001) 381.

[8] STROTH, U., et al., Nucl.Fusion 36 (1996) 1063.

[9] YAMADA, H., et al., Plasma Phys. Control. Fusion 43 (2001) A55.

[10] SUDO, S., et al., Nucl. Fusion 30 (1990) 11.

[11] SAKAKIBARA, S., et al., Plasma Phys. Control. Fusion 44 (2002) A217.

[12] NAKAJIMA, N., et al., Nucl. Fusion 46 (2006) 177.

[13] YAMADA, H., et al, Nucl. Fusion 45 (2005) 1684.

\section{LHD Experimental Group}

T.Akiyama, N.Ashikawa, H.Chikaraishi, M.Emoto, N.Ezumi 1), H.Funaba, P.Goncharov, M.Goto, Y.Hamada, S.Hamaguchi, Y.Hatano 2), T.Hino 3), M.Hoshino 4), K.Ichiguchi, M.Ichimura 5), K.Ida, H.Idei 6), T.Ido, H.Igami, Y.Igitkanov 22), K.Ikeda, S.Imagawa, S.Inagaki, K.Irie 7), K.Ishii 5), 
A.Isayama 8), M.Isobe, K.Itoh, A.Iwamoto, S.Kado 9), D.Kalinina, Y.Kamada 8), O.Kaneko, D.Kato, T.Kato, K.Kawahata, M.Kobayashi, S.Kobayashi 10), T.Kobuchi, A.Komori, K.Kondo 11), S.Kubo, R.Kumazawa, R.Maekawa, S.Masuzaki, O.Mitarai 12), T.Mito, J.Miyazawa, T.Morisaki, S.Morita, O.Motojima, I.Murakami, S.Murakami 13), S.Mutoh, T.Mutoh, K.Nagaoka, K.Nagasaki 10), Y.Nagayama, Y.Nakajima, Y.Nakamura, H.Nakanishi, K.Narihara, Y.Narushima, K.Nishimura, H.Nishimura 14), N.Nishino 15), M.Nishiura, N.Noda, T.Notake, H.Ogawa 16), S.Ohdachi, N.Ohno 17), N.Ohyabu, Y.Oka, H.Okada 10), T.Okamura 18), K.Okuno 19), K.Ono 20), M.Osakabe, T.Ozaki, R.Pavlichenko, B.J.Peterson, A.Sagara, K.Saito, T.Saito 5), S.Sakakibara, M.Sakamoto 6), R.Sakamoto, M.Sasao 21), K.Sato, T.Seki, A.Shimizu, T.Shimozuma, M.Shiratani 6), M.Shoji, S.Sudo, H.Sugama, C.Suzuki, Y.Suzuki, K.Takahata, S.Takamura 4), Y.Takeiri, H.Takenaga 8), H.Tamura,N.Tamura, K.Tanaka, K.Toi, M.Tokitani 6), T.Tokuzawa, Y.Torii 10), H.Tsuchiya 16), K.Tsumori, F.Watanabe 4), A.Weller 22), E.Veshchev 16), L.N.Vyacheslavov 23), K.Y.Watanabe, H.Yamada, I.Yamada, S.Yamada, N.Yanagi, O.Yamagishi, S.Yamaguchi, T.Yamaguchi, M.Yokoyama, N.Yoshida 6), M.Yoshinuma, S.Yoshimura, Y.Yoshimura

National Institute for Fusion Science, Toki, Gifu, Japan

1) Nagano National College of Technology, Nagano, Japan

2) Hydrogen Isotope Research Center, Toyama Univ., Toyama, Japan

3) Graduate School of Engineering, Hokkaido Univ., Sapporo, Japan

4) Graduate School of Engineering, Nagoya Univ., Nagoya, Japan

5) Plasma Research Center, Tsukuba Univ., Tsukuba, Ibaraki, Japan

6) Research Institute of Applied Mechanics, Kyushu Univ., Kasuga, Fukuoka, Japan

7) School of Science and Engineering, Waseda Univ., Tokyo, Japan

8) Naka Fusion Institute, Japan Atomic Energy Agency, Naka, Ibaraki, Japan

9) High Temperature Plasma Center, The Univ. of Tokyo, Tokyo, Japan

10) Institute of Advanced Energy, Kyoto Univ., Uji, Kyoto, Japan

11) Graduate School of Energy Science, Kyoto Univ., Uji, Japan

12) Graduate School of Engineering, Kyushu Tokai Univ., Kumamoto, Japan

13) Graduate School of Engineering, Kyoto Univ., Kyoto, Japan

14) Institute of Laser Engineering, Osaka Univ., Suita, Japan

15) Graduate School of Engineering, Hiroshima Univ., Higashihiroshima, Japan

16) School of Physical Science, The Graduate University for Advanced Studies, Hayama, Japan

17) EcoTopia Science Institute, Nagoya Univ., Nagoya, Aichi, Japan

18) Interdisciplinary Graduate School of Science and Engineering, Tokyo Institute of Technology, Tokyo, Japan

19) Radiochemistry Research Lab., Shizuoka Univ., Shizuoka, Japan

20) Interdisciplinary Faculty of Science and Engineering, Shimane Univ., Matsue, Japan

21) Graduate School of Engineering, Tohoku Univ., Sendai, Japan

22) Max-Planck-Institut fuer Plasmaphysik, Germany

23) Budker Institute of Nuclear Physics, Russia 